\title{
MOA Duplication \& Deletion Theory: COVID-19 Pathogenesis Mechanism: Gene Duplication, Retrotransposition, 4p15.1-pter Duplication and a 4q35.1-qter Deletion in a Recombinant of Chromosome 4 Pericentric Inversion
}

\author{
Mosab Nouraldein Mohammed Hamad \\ Research Unit, Banoon Fertility Center, Sudan
}

DOI: $10.36348 /$ sjbr.2020.v05i04.002 $\quad$ | Received: 07.04.2020 | Accepted: 18.04.2020 | Published: 22.04 .2020

*Corresponding author: Mosab Nouraldein Mohammed Hamad

\section{Abstract}

Coronavirus disease 2019 (COVID-19) is an infectious disease caused by severe acute respiratory syndrome Coronavirus 2 (SARS-CoV-2). It is affecting 213 countries and territories around the world. The disease causes respiratory illness (like the flu) with symptoms such as a cough, fever, and in more severe cases, difficulty breathing, cardiac problems were also observed in many COVID-19 patients. We theorize that COVID-19 induce mutation in chromosome 4 lead to 4p15.1-pter Duplication and a 4q35.1-qter Deletion, and the neurological and cardiac disorders observed among infected persons resulted from retrotransposition mechanism of gene duplication occur in the specified region of chromosome 4 mentioned above. Genetic studies to chromosome 4 of COVID -19 patients recommended to confirm MOA duplication and deletion theory (4p15.1-pter Duplication and a 4q35.1-qter Deletion).

Keywords: COVID-19pathogensis mechanism, Chromosome 4, Gene duplication, Retrotransposition.

Copyright @ 2020: This is an open-access article distributed under the terms of the Creative Commons Attribution license which permits unrestricted use, distribution, and reproduction in any medium for non-commercial use (NonCommercial, or CC-BY-NC) provided the original author and source are credited.

\section{BACKGROUND}

Coronaviruses are a family of enveloped RNA viruses that are spread broadly among mammals and birds, causing chiefly respiratory or enteric illnesses but in some cases neurologic disease or hepatitis. Individual coronaviruses typically infect their hosts in a speciesspecific mode, and infections acute or constant. Infections transmitted mostly via respiratory and fecaloral routes. The most characteristic character of this viral family is genome size: coronaviruses have the largest genomes among all RNA viruses, counting those RNA viruses with segmented genomes [1].

In winter 2019, a group of patients admitted to hospitals with an early diagnosis of pneumonia of an unidentified etiology. These infected persons were epidemiologically connected to a seafood and wet animal wholesale marketplace in Wuhan, China [2]. CVID-19 outbreak disseminated in 213 countries with 1.954.724 confirmed cases and 126.140 confirmed deaths [3].

While COVID-19 is mainly a respiratory infection, it has significant systemic consequences counting on the cardiovascular and immune systems.
Patients with pre-existing cardiovascular disorders represent big proportions of patients with symptomatic infection, and experience disproportionately poorer outcomes at between 5-10 fold raise in mortality. Patients with new COVID-19 infections can also expand cardiovascular difficulties, such as heart failure, myocarditis, pericarditis, vasculitis, and cardiac arrhythmias. Among 8-28\% of patients with COVID-19 infections will obvious troponin discharge early in the course of the disease, indicating cardiac injury or stress.

SARS-Cov-2 has a polybasic (furin) cleavage position inserted at the border of the $\mathrm{S} 1 / \mathrm{S} 2$ subunits of the spike S-protein. This furin linking place is unique, can promote the virus' capability to internalize into cells [4].

Gene duplication is an event in which one gene gives rise to two genes that cannot be operationally notable from each other. The duplicated genes stay in the same genome and so are paralogues and in dissimilar genome as orthologues. Gene duplication supposed to play a significant role in evolution by providing material for evolution of novel gene functions. Duplicated genes shaped by unequal 
crossing over, retrotransposition, duplicated DNA transposition and polyploidization [5].

Vertebrate genomes hold thousands of pseudo genes extends of DNA sequence with homology to useful genes. Pseudogenes create through an assortment of methods counting retrotransposition of processed mRNAs (processed pseudogenes), segmental duplication, and inactivating mutations. Processed pseudogenes are resulting from spliced transcripts and they lack the intron-exon structure of their source gene.

Retrotransposition refers to insertion of DNA sequences arbitrated by an RNA intermediate. In humans, this method passed out chiefly through the reverse-transcriptase and endonuclease functions of the LINE-1 ORF2 protein, with support from the ORF1 protein, which links RNA and functions as a chaperone [6].This DNA polymerase at that point duplicates the RNA into DNA. That cDNA duplicate of the retrotransposon changed to a twofold stranded item and embedded at a stunned part at the target place. The enzymes needed for amalgamation the reverse transcript (first strand of the new copy) to the other end of the staggered split and for second strand synthesis have not yet been recognized [7]. LINE-1(L1) elements are the merely active and autonomous transposable elements in humans. The center retrotransposition apparatus is a ribonucleoprotein particle (RNP) holding the L1 mRNA, with endonuclease and reverse transcriptase activities. It starts reverse transcription directly at genomic aim positions upon endonuclease cleavage.

Transposable components account for half to two-thirds of the human genome. Among them, LINE-1 (L1) non-LTR retrotransposons form the merely autonomous and dynamic family and are the most copious, representing $17 \%$ of our DNA. L1 insertions are essentially mutagenic; though their real impact on gene expression depends on their specific place of integration. Intergenic or deep intronic insertions can often have no detectable influences on genes. In contrast, insertions in exons or regulatory sequences have the potential to intensely change gene expression/function, by upsetting coding- or cisregulating sequences, or by transport cis-regulating sequences (transcription factor binding sites, cryptic splicing and polyadenylation sites, etc.). Therefore, germline L1 insertions sporadically lead to de novo genetic diseases.

The $\mathrm{L} 1$ replication cycle begins with union of bicistronic mRNA coding for the twoL1 Proteins, ORF1p and ORF2p. ORF1p is a $40 \mathrm{kDa}$ RNA-binding protein capable to form trimers. It reveals nucleic acid chaperone activity, the function of which has not been elucidated. ORF2p may be a huge, $149 \mathrm{KDa}$, protein with endonuclease (EN) and turn around transcriptase (RT) actions. Both ORF1p and ORF2p tie the L1 RNA to make steady ribonucleoprotein molecule (RNP), the center of the L1 RNP can intervene two diverse integration forms. In the canonical pathway, called target-primed reverse transcription (TPRT), the L1 EN activity produces a nick at the recognized target site in the chromosomal DNA. The RT moiety then extends this released 3 '-OH group, using the L1 RNA as a template. Reverse transcription prepared inside the poly (A) tail of the L1 RNA. L1 EN preferentially cuts DNA at a consensus sequence 5'-TTTTA-3', with nicking occurring at the TpA bond [8].

Pericentric inversions are noticed with unstable frequency in all human chromosomes. Breakpoint regions of chromosomal inversions often hold high densities of repetitive DNA sequences, such as Alu and L1 elements, leading to speculation that they could arbitrate chromosomal rearrangements and act as hot spots for non-allelic homologous recombination (NAHR).

The chromosome 4 inversion engaging subband p14 p15 and $\mathrm{q} 35$ results in two kinds of recombinant chromosome 4 . About $80 \%$ of the practical recombinants are partial $4 \mathrm{p}$ duplications and $4 \mathrm{q}$ deletions [9].

Study done by Rong Yin showed that; Neurological assessment indicated that COVID-19 confirmed patient was in a poor mental state, with his consciousness changing between lethargy and irritability. His responses to questions were wrong, with some signs of contextually dissociated speech [10], another study conducted by Maurin M-L et al. showed that; Duplications of the distal $2 / 3$ of short arm of chromosome 4 (4p15.1-pter duplication) associated with mental retardation, while a 4q35.1-qter Deletion in the same chromosome combined with cardiac defects[11].

\section{MOA Duplication \& Deletion Theory}

The pathogenesis of COVID-19 attributed to its ability to induce 4p15.1-pter duplication and a 4q35.1-qter deletion in a recombinant of chromosome-4 pericentric inversion through gene duplication method named retrotransposition, duplication lead to mental defects, while deletion lead to cardiac defects due to loss of ArgBP2 and PDLIM3 genes, whom are necessary for normal cardiac muscle functions.

\section{CONCLUSION}

Our theory attribute all clinical features and complications accompanied with COVI-D 19 infection to the 4p15.1-pter duplication and 4q35.1-qter Deletion induced by SARS-cov-2.

\section{RECOMMENDATIONS}

Genetics studies required for COVID-19 patients to check chromosome 4, mainly 4p15.1-pter and $4 \mathrm{q} 35.1$-qter regions to comment on the theory. 


\section{REFERENCES}

1. Masters, P. S. (2006). The molecular biology of coronaviruses. Advances in virus research, 66, 193-292.

2. World Health Organization. (2019). Coronavirus disease (COVID-19) outbreak situation, https://www.who.int/emergencies/diseases/novelcoronavirus-2019.

3. Rothan HA, Byrareddy SN. The epidemiology and pathogenesis of coronavirus disease (COVID-19) outbreak. J Autoimmun. 2020; 109:102433.

4. Liu, P. P., Blet, A., Smyth, D., \& Li, H. (2020). The Science Underlying COVID-19: Implications for the Cardiovascular System. Circulation.

5. Santoshkumarmagadum, Urbibanerjee, Priyadharshinimurugan, Doddabhimappagangapur \& Rajasekarravikesavan. (2013). Gene duplication as a major force in evolution, Journal of Genetics, 92(1): 155-161.

6. Ewing, A. D., Ballinger, T. J., Earl, D., Harris, C. C., Ding, L., Wilson, R. K., \& Haussler, D. (2013). Retrotransposition of gene transcripts leads to structural variation in mammalian genomes. Genome biology, 14(3), R22.

7. Ross, H. Mechanism of Retrotransposition, https://bio.libretexts.org/Bookshelves/Genetics/Boo k\%3A_Working_with_Molecular_Genetics_(Hardi
son)/Unit_II\%3A_Replication\%2C_Maintenance_a nd_Alteration_of_the_Genetic_Material/9._Transp osition_of_DNA/Mechanism_of_Retrotranspositio n.

8. Viollet, S., Monot, C., \& Cristofari, G. (2014). L1 retrotransposition: the snap-velcro model and its consequences. Mobile genetic elements, 4(2), e1003499.

9. Hemmat, M., Hemmat, O., Anguiano, A., Boyar, F. Z., El Naggar, M., Wang, J. C., ... \& Haddadin, M. (2013). Genotype-phenotype analysis of recombinant chromosome 4 syndrome: an arrayCGH study and literature review. Molecular cytogenetics, 6(1), 17.

10. Yin, R., Feng, W., Wang, T., Chen, G., Wu, T., Chen, D., \& Xiang, D. (2020). Concomitant neurological symptoms observed in a patient diagnosed with coronavirus disease 2019. Journal of Medical Virology.

11. Maurin, M. L., Labrune, P., Brisset, S., Le Lorc'h, M., Pineau, D., Castel, C., \& Tachdjian, G. (2009). Molecular cytogenetic characterization of a $4 \mathrm{p} 15$. 1- pter duplication and a 4q35. 1- qter deletion in a recombinant of chromosome 4 pericentric inversion. American Journal of Medical Genetics Part A, 149(2), 226-231. 